\title{
Idosos vítimas de trauma: doenças preexistentes, medicamentos em uso no domicílio e índices de trauma
}

\author{
Elderly victims of trauma: preexisting conditions, medications taken at home and indexes of trauma
}

Ancianos víctimas de trauma: condiciones preexistentes, medicamentos tomados en el hogar e índices de trauma

\section{Gláucia Costa Degani', Gerson Alves Pereira Júnior", Rosalina Aparecida Partezani Rodrigues', Bruna Moretti Luchesi', Sueli Marques'}

\begin{abstract}
' Universidade de São Paulo, Escola de Enfermagem de Ribeirão Preto, Programa de Pós-Graduação em Enfermagem. Ribeirão Preto-SP, Brasil.

"Universidade de São Paulo, Faculdade de Medicina de Ribeirão Preto,

Departamento de Cirurgia e Anatomia. Ribeirão Preto-SP, Brasil.
\end{abstract}

Submissão: 19-04-2013 Aprovação: 21-08-2014

\section{RESUMO}

Objetivou-se identificar o perfil sociodemográfico de idosos vítimas de trauma, caracterizar doenças preexistentes e medicamentos utilizados no domicílio; calcular índices de trauma e desfecho clínico. Estudo retrospectivo e exploratório, com a análise de dados secundários de um banco de dados de um hospital geral terciário, entre 2008 e 2010 . Foram estudados 131 idosos, média de idade 69,9 anos, 73,3\% homens, 55,1\% casados, 54,7\% aposentados; 65,6\% possuíam doenças preexistentes e 48,9\% usavam medicamentos no domicílio. Houve representatividade de quedas (31,3\%), seguidas por atropelamento (28,2\%), com cabeça/pescoço sendo a região mais acometida (59,5\%). Prevaleceu o trauma moderado $(44,3 \%)$, com condições de sobrevida após o evento $(80,2 \%)$. Houve associação entre mecanismo do trauma e doença preexistente $(p=0,01)$ e entre mecanismo do trauma e sexo $(p=0,03)$. O conhecimento das variáveis envolvidas com idosos vítimas de trauma possibilita aos profissionais de saúde o planejamento de medidas preventivas, visando aprimorar sua assistência.

Descritores: Idoso; Ferimentos e Lesões; Doença; Uso de Medicamentos.

\section{ABSTRACT}

The objective was to identify the sociodemographic profile of the elderly victims of trauma, to characterize preexisting conditions and medications taken at home, and to calculate indices of trauma and clinical outcomes. This is a retrospective and exploratory analysis from a database of a general hospital between 2008 and 2010. There were studied 131 elderly, mean age 69.9 years, $73.3 \%$ male, $55.1 \%$ married, $54.7 \%$ retired, $65.6 \%$ had preexisting conditions and $48.9 \%$ used drugs at home. There was a representative number of falls $(31.3 \%)$, followed by running over $(28.2 \%)$, with the head/neck region being the most affected $(59.5 \%)$. Moderate trauma prevailed $(44.3 \%)$, with conditions of survival after the event $(80.2 \%)$. There was an association between mechanism of trauma and preexisting disease $(p=0.01)$ and between mechanism of trauma and sex $(p=0.03)$. The knowledge of the variables involved with the elderly victims of trauma enables healthcare professionals to plan preventive measures aimed at improving the assistance.

Key words: Aged; Wounds and Injuries; Disease; Drug Utilization.

\section{RESUMEN}

Se objetivó identificar el perfil sociodemográfico de ancianos víctimas de trauma, caracterizar condiciones preexistentes y medicamentos tomados en casa, y calcular índices de trauma y evolución clínica. Se realizó un análisis retrospectivo y exploratorio de una base de datos de un hospital general terciario entre 2008 y 2010 . Se estudiaron 131 ancianos, media of 69,9 años, $73,3 \%$ hombres, 55,1\% casados, 54,7\% jubilados, 65,6\% tienen condiciones preexistentes y 48,9\% estaban tomando medicación en casa. Hubo representación de las caídas (31,3\%), seguido de atropello (28,2\%). La región cabeza/cuello fue el más afectado (59,5\%). Prevaleció trauma moderado (44,3\%), con condiciones de supervivencia después del evento (80,2\%). Se observó una asociación entre mecanismo de lo trauma y enfermedad previa $(p=0,01)$ y entre mecanismo de lo trauma y sexo $(p=0,03)$. El conocimiento de las variables que intervienen con ancianos víctimas de trauma permite a los profesionales de la salud planificar medidas preventivas para mejorar su asistencia.

Palabras claves: Anciano; Heridas y Traumatismos; Enfermedad; Utilización de Medicamentos.

$$
\text { AUTOR CORRESPONDENTEＳueli Marques E-mail: smarques@eerp.usp.br }
$$




\section{INTRODUÇÃO}

Assim como em países desenvolvidos, o Brasil também passa por uma transição demográfica, pois se observa um significativo aumento de pessoas com 60 anos ou mais em relação às demais faixas etárias. Essas pessoas, denominadas idosos, possuem participação ainda ativa na sociedade, resultado dos constantes esforços em promover uma forma de vida mais saudável ${ }^{(1)}$ e, também a busca pessoal de novas experiências culturais e recreativas. Este estilo de vida ocorre, em sua maioria, em um cenário urbano onde, a falta de acessibilidade nos espaços domésticos e públicos, em particular de vias públicas, expõe os idosos ao risco de acidentes e violências.

Os traumas repercutem como um evento com representatividade para esta população, pois, muitas vezes, prejudicam sua capacidade física e mental ${ }^{(2)}$, trazem importantes consequências sociais e econômicas, e são responsáveis por altos coeficientes de mortalidade. No Brasil, no ano de 2011, dados estatísticos relacionados às internações hospitalares por causas externas mostram que essas foram representadas por 6,44\%, ou seja, 160.781 internações no Sistema Único de Saúde, segundo capítulos da Classificação Internacional de Doenças (CID10), com expectativa de aumento com o passar dos $\operatorname{anos}^{(3)}$.

Trauma define-se como o termo médico cujo significado é lesão; deve ser considerado uma doença e não um acidente ou fatalidade e, assim, pode-se evitá-lo. Os idosos são expostos aos mesmos mecanismos de trauma que a população geral, a diferença está na dimensão em que eles ocorrem ${ }^{(4)}$. O trauma em idosos possui características específicas e alguns fatores, como por exemplo, as alterações decorrentes do próprio processo de envelhecimento, a associação à doenças preexistentes e o uso concomitante de medicamentos podem interferir negativamente em sua morbimortalidade.

Os efeitos do envelhecimento nos sistemas orgânicos, os quais podem ser descritos por sua progressiva perda funcional, ao tentarem responder às exigências do trauma, encontram outras complicações ${ }^{(4)}$. Associado a isso, é comum que idosos apresentem ao menos uma doença prévia, o que corresponde, por si só, a um fator preditivo da mortalidade em vítimas de trauma ${ }^{(5)}$. Dentre elas, é comum encontrar na população geral o diabetes mellitus, a hipertensão arterial sistêmica, coronariopatia, insuficiência cardíaca, doença pulmonar obstrutiva crônica, insuficiência renal crônica e cirrose hepáti$\mathrm{ca}^{(4)}$. Sendo assim, habitualmente, os idosos fazem uso de um ou mais medicamentos para controle de doenças, como por exemplo, os psicotrópicos (antidepressivos, sedativos, neurolépticos), que estão associados com a ocorrência de quedas; os anti-hipertensivos, que podem tornar a ressuscitação difícil após o trauma; os beta-bloqueadores que, especificamente, podem diminuir a resposta hemodinâmica compensatória à perda de volume ou sangue; e os anticoagulantes e agentes antiplaquetários, que aumentam o risco de complicações hemorrágicas $^{(6)}$. Portanto, ao realizar o atendimento ao idoso, vítima de trauma, deve-se ter em mente estas variáveis.

Os índices de trauma possibilitam quantificar as alterações fisiológicas e a gravidade das lesões anatômicas apresentadas pelas vítimas e calcular a probabilidade de sobrevida, contribuindo na avaliação da qualidade do atendimento prestado ${ }^{(7)}$. Diversos índices fisiológicos e anatômicos têm mostrado relação com o trauma geriátrico, destacando-se: Revised Trauma Score (RTS), Injury Severity Score (ISS) e Trauma and Injury Severity Score (TRISS) ${ }^{(6)}$.

Este estudo justifica-se à medida que se pretende reconhecer em idosos, vítimas de trauma com maior gravidade, a presença de variáveis que podem ser responsáveis pelo prolongamento da permanência hospitalar, pelo surgimento de complicações, incapacidades e mortes. Além disso, pesquisas nesta temática, especialmente com a utilização dos índices de trauma, ainda são incipientes no país.

O objetivo foi identificar o perfil sociodemográfico de idosos, vítimas de trauma; caracterizar as doenças preexistentes e os medicamentos em uso no domicílio; calcular os índices de trauma; descrever o desfecho clínico e verificar a associação das variáveis faixa etária, sexo e mecanismo do trauma com as variáveis doenças, mecanismo e os índices de trauma.

\section{METODOLOGIA}

Estudo retrospectivo e exploratório, realizado a partir da análise dos dados de natureza secundária contidos no banco de dados de vigilância das causas externas do Núcleo Hospitalar de Epidemiologia (NHE) de um hospital geral terciário do interior paulista, referentes às notificações dos pacientes, vítimas de trauma, no período de janeiro de 2008 a dezembro de 2010.

Os critérios de inclusão do estudo foram: possuir 60 anos ou mais de idade, ambos os sexos, vítimas de trauma, que foram atendidos na Sala de Trauma, cadastrados no banco de dados do NHE. Idosos com fratura isolada de colo de fêmur pós-queda da própria altura e aqueles com lesões ortopédicas com ISS $\leq 8$ pontos foram excluídos, pois atualmente estudos se dedicam a compreender a queda da própria altura e suas consequências ${ }^{(8)}$, além do que a mesma possui baixa gravida$\mathrm{de}^{(9)}$. Ao excluí-la, pretende-se evidenciar os traumas de gravidade moderada a muito grave (ISS $\geq 9$ ).

A população total de vítimas de trauma, cadastradas no referido banco nos três anos analisados foi de 5.945 pacientes, destes, 470 (7,9\%) eram idosos. Após a aplicação dos critérios de inclusão/exclusão, a amostra deste estudo foi constituída por 131 idosos.

A coleta de dados seguiu as informações contidas no banco de dados do NHE, considerando seu sistema de medidas: 1) sociodemográficas - idade, sexo, estado civil e profissão/ ocupação; 2) características do evento traumático - tipo de transporte pré-hospitalar e mecanismo do trauma; 3) índices de trauma - Revised Trauma Score (RTS), Abreviated Injury Scale (AIS), Injury Severity Score (ISS) e Trauma and Injury Severity Score (TRISS); e fator de alteração de consciência (sob efeito de álcool, sedado, sedado e entubado, epiléptico e outros); 4) doenças pré-existentes e uso de medicamentos em domicílio e 5) evolução clínica - necessidade de cirurgia e qual especialidade, necessidade de internação na Unidade de Terapia Intensiva (UTI), tempo de internação e, no óbito, qual a causa principal. 
O RTS é um índice fisiológico que possibilita avaliar a resposta orgânica ao trauma a partir de parâmetros da avaliação do estado neurológico pela Escala de Coma de Glasgow $(\mathrm{ECGl})$, da pressão arterial sistólica e da frequência respiratória; os quais são determinados no momento da admissão hospitalar, podendo estar alterados se o paciente estiver sob efeito anestésico, álcool, drogas ilícitas ou se foi entubado ${ }^{(10)}$. Quanto maior a pontuação, maiores as chances de sobrevida.

A AIS contém uma lista de milhares de lesões com valores de gravidade que variam de 1 a 6 , dividindo-se o corpo em seis regiões: cabeça e pescoço, face, tórax, abdome e órgãos da pelve, extremidades e ossos da pelve e superfície externa. A gravidade das lesões é classificada em: 1 - gravidade mínima; 2 - moderada; 3 - grave, sem risco de vida; 4 - grave, com risco de vida, mas de provável sobrevida; 5 - crítica, sobrevivência duvidosa e 6 - quase sempre fatal ${ }^{(10)}$.

O ISS é um índice anatômico, considerando a gravidade das lesões existentes nos seguimentos do corpo. É baseado na AIS e, portanto, seu cálculo é realizado de acordo com os índices mais graves de cada uma das seis regiões do corpo, assim, o ISS pode variar de 1 a 75 pontos $^{(7)}$. Neste estudo, os valores do ISS obtidos foram categorizados de 9 a 15 pontos (trauma moderado), 16 a 24 (trauma grave) e 25 pontos ou mais (trauma muito grave) ${ }^{(10)}$.

Já o TRISS, escore de trauma e o escore de gravidade da lesão, é um índice que associa o RTS e o ISS, considerando-se, ainda, a idade e o tipo de trauma (contuso ou penetrante), possibilitando o cálculo da probabilidade de sobrevida. Pode ser interpretado como: de $0-25 \%$, associado à inevitabilidade da morte; entre $26-50 \%$, significa indivíduos potencialmente salváveis; e 51\% ou mais, indica francas condições de se evitar o óbito ${ }^{(10)}$.

Obteve-se um check-list das doenças preexistentes e medicamentos em uso procedentes dos prontuários médicos, a partir da elaboração e validação de um instrumento de coleta de dados, que posteriormente, foram agrupados conforme os capítulos da CID10 e de acordo com o Dicionário de Especialidades Farmacêuticas, respectivamente.

Para o processamento dos dados, foi elaborada uma planiIha em Microsoft Excel, contendo um dicionário (codebook) e duas planilhas que foram utilizadas para a validação por dupla entrada (digitação). Após, os dados foram exportados para o software estatístico SAS ${ }^{\circledast}$ 9.0, utilizado para realizar todas as análises estatísticas. Os dados foram interpretados de acordo com estatísticas descritivas - frequência simples, medida de tendência central - média (méd), de variabilidade - desvio padrão (dp) e correlacionais. Valores de p menores que 0.05 foram considerados significativos.

Para a análise da associação entre as variáveis faixa etária, sexo e mecanismo do trauma com as variáveis doença, ISS e mecanismo do trauma foram realizados alguns agrupamentos. Foram considerados: acidentes de transporte (códigos do trauma referentes aos pedestres, ciclistas, veículos automotores e motociclistas), quedas, queimadura e violência (agressão, ferimento por arma de fogo e arma branca).

O projeto foi apreciado pelo Comitê de Ética em Pesquisa da Escola de Enfermagem de Ribeirão Preto, sendo aprovado, sob o protocolo n ${ }^{\circ} 1261 / 2011$.

\section{RESULTADOS}

\section{Perfil sociodemográfico}

A idade dos idosos variou entre 60 e 93 anos (méd $=69,9$ anos; $d p=7,7$ anos). $O$ intervalo com maior distribuição foi o de 60-69 anos com 77 (58,8\%) participantes de ambos os sexos. Houve o predomínio do sexo masculino 96 (73,3\%) e, com relação ao estado conjugal, a maioria era casada/amasiada $71(55,1 \%)$ e $70(54,7 \%)$ eram aposentados ou pensionistas.

\section{Doenças preexistentes e medicamentos em uso no domicílio}

A maior parte dos idosos $(65,6 \%)$ apresentava doenças preexistentes, sendo que $58,0 \%$ possuíam alguma doença do aparelho circulatório, 49,4\% transtornos mentais e comportamentais e $26,6 \%$, doenças endócrinas, nutricionais e metabólicas. Vale ressaltar que um mesmo idoso poderia estar presente em uma ou mais categoria ao mesmo tempo. Destaca-se, dentre elas, a hipertensão arterial sistêmica (HAS) 51 $(38,9 \%)$, o etilismo $26(19,8 \%)$ e o tabagismo $19(14,4 \%)$ e o diabetes mellitus tipo $219(14,4 \%)$, respectivamente.

O número de doenças preexistentes por idoso variou entre zero e seis (méd $=2,3 ; d p=1,4)$, sendo que $33(25,2 \%)$ possuíam uma doença, $20(15,3 \%)$, duas e $17(13,0 \%)$, três. Ressalta-se que para $21(16,0 \%)$ deles havia, em seus prontuários médicos, a informação de que não possuíam qualquer doença e para $24(18,3 \%)$ não havia registro se possuíam ou não.

Houve o predomínio do uso de medicamentos em domicílio $(48,9 \%)$, destes, $33,4 \%$ faziam uso de anti-hipertensivos, $19,8 \%$ de diuréticos e $11,4 \%$ de antiagregante plaquetário/ anticoagulante. Destaca-se que um mesmo idoso poderia estar presente em uma ou mais categorias ao mesmo tempo. Na categoria de anti-hipertensivos, os inibidores da enzima conversora da angiotensina eram usados por $38(28,9 \%)$ dos idosos e os betabloqueadores cardiosseletivos, por $22(16,7 \%)$.

$\mathrm{O}$ número de medicamentos em uso pelos idosos variou entre zero e nove (méd $=3,2 ; d p=2,3$ ). Nota-se que 22 $(16,8 \%)$ tomavam um medicamento, $10(7,6 \%)$, dois e 10 $(7,6 \%)$, três.

\section{Características do trauma}

Quanto ao tipo de transporte pré-hospitalar, $36(27,5 \%)$ idosos foram transportados pelas Unidades de Suporte Avançado (USAs) de Ribeirão Preto, seguido de $31(23,7 \%)$ pelas Unidades de Suporte Básicos (USBs) de outras cidades e 30 $(22,9 \%)$ pelas USBs de Ribeirão Preto.

Constatou-se que, para $41(31,3 \%)$ idosos, o mecanismo de trauma foi a queda, seguido de $37(28,2 \%)$ atropelamento e $17(13,0 \%)$ para as queimaduras. Em relação ao tipo de trauma, $109(83,2 \%)$ idosos apresentaram trauma contuso. De todos os traumas, sete $(5,3 \%)$ foram caracterizados como tentativa de autoextermínio.

Considerando as regiões do corpo afetadas, nota-se que $78(59,5 \%)$ idosos apresentaram alguma lesão na região da cabeça/pescoço, seguida por $60(45,8 \%)$ na região das extremidades e ossos da pelve. Dentre os 78 idosos com lesão na região da cabeça/pescoço, 43,6\% foram classificados com 
AIS 3 (grave, sem risco de vida) e para os com lesão na região das extremidades e ossos da pelve (60), em 16,7\% o AIS correspondeu a 3 (grave, sem risco de vida).

O número de lesões por idoso variou entre uma e seis (méd $=1,8 ; d p=1,1)$. No que se refere à relação entre lesões e região do corpo, 69 (52,7\%) apresentaram lesões em apenas uma região do corpo, $34(26,0 \%)$ em duas regiões, 19 $(14,5 \%)$ em três e 9 (6,9\%) em quatro ou mais regiões.

Tabela 1 - Distribuição dos idosos, vítimas de trauma, nos anos 2008, 2009 e 2010, segundo os índices de trauma RTS, ISS e TRISS, Ribeirão Preto-SP, 2011

\begin{tabular}{lrr}
\hline Variáveis & $\mathbf{n}$ & $\%$ \\
\hline Escore de trauma revisado - RTS (PONTOS) & & \\
8 & 83 & 63,4 \\
7 & 16 & 12,2 \\
6 & 13 & 9,9 \\
5 & 5 & 3,8 \\
4 & 7 & 5,3 \\
3 & 4 & 3,1 \\
0 & 2 & 1,5 \\
Ignorado & 1 & 0,8 \\
Índice de gravidade da lesão - ISS (PONTOS) & & \\
9 a 15 (trauma moderado) & 58 & 44,3 \\
16 a 24 (trauma grave) & 33 & 25,2 \\
25 ou mais (trauma muito grave) & 40 & 30,5 \\
Escore de trauma e o escore & & \\
de gravidade da lesão - TRISS (\%) & & \\
0 a 25 & 12 & 9,2 \\
26 a 50 & 13 & 9,9 \\
51 a 75 & 8 & 6,1 \\
76 a 90 & 25 & 19,1 \\
91 a 95 & 31 & 23,7 \\
96 a 100 & 41 & 31,3 \\
Ignorado & 1 & 0,8 \\
& & \\
\hline
\end{tabular}

De acordo com a Tabela 1, a pontuação do RTS obteve méd $=7,0$ e $d p=1,6$; sendo que $63,4 \%$ deles apresentaram escores aproximados de 8 , ou seja, alta probabilidade de sobrevida. Quanto ao ISS, houve méd =19,7 e $d p=13,8$. Nota-se que $44,3 \%$ apresentaram trauma moderado, seguidos de trauma muito grave $(30,5 \%)$ e trauma grave $(25,2 \%)$. Quanto ao TRISS, a pontuação variou entre 0,2 e $97 \%$, com média de $78,1 \%$, sendo que $80,2 \%$ apresentaram valores de $51 \%$ ou mais, o que indica franca condições de se evitar o óbito. Vale ressaltar que, na admissão hospitalar, dados da avaliação inicial revelaram que 109 (83,2\%) das vítimas não apresentavam nenhum fator de alteração da consciência.

Foi possível verificar a associação entre mecanismo do trauma e doença preexistente $(p=0,01)$, sendo que $33(31,4 \%)$ daqueles com doença preexistente foram vítimas de acidente de transporte, seguida de 31 (29,5\%) por quedas. Houve, também, associação entre mecanismo do trauma e sexo $(p=0,03)$, com predomínio de acidentes entre os homens, 52 (40,6\%) e as quedas entre as mulheres, 14 (10,9\%).

Verificou-se que os acidentes foram o mecanismo do trauma que mais acometeram idosos na faixa etária entre 60-69 anos representados por 36 (28,1\%); entre $70-79$ anos por 18 $(14,1 \%)$; entre aqueles com 80 ou mais, prevaleceram as quedas, por $10(7,8 \%)$.

Quanto ao desfecho clínico do trauma, $86(65,6 \%)$ idosos necessitaram realizar algum procedimento cirúrgico, com destaque para o ortopédico em 39 (46,1\%). O número de dias de internação variou entre zero e 116 dias, sendo que 57 $(43,5 \%)$ permaneceram entre um e 10 dias internados. Dos idosos vítimas de trauma, 44 (33,6\%) evoluíram a óbito, apresentando como principal causa o traumatismo crânio-encefálico para $16(36,4 \%)$.

\section{DISCUSSÃO}

Possivelmente, a média de idade encontrada neste estudo deve-se ao fato dos idosos ainda apresentarem um perfil de população adulta, ou seja, com independência e autonomia, o que Ihes proporciona vida ativa com participação em atividades sociais, de trabalho, de lazer, entre outras que podem expô-lo a situações de trauma.

Supõe-se que o predomínio do sexo masculino e da média de idade dos idosos pesquisados tenha sido influenciado pelos critérios de seleção e, especialmente, pelos de exclusão. Provavelmente, homens nas faixas etárias mais novas, se exponham mais a traumas, pelo fato de que circulam mais pelo perímetro urbano, enquanto que as idosas, na maioria das vezes, circulam por lugares mais próximos de seus domicílios ${ }^{(11)}$ Ainda chama a atenção que as práticas de risco estejam fortemente associadas ao sexo masculino, o que torna os homens as maiores vítimas da violência, potencializada pelas desigualdades sociais e outras condições adversas ${ }^{(12)}$.

Em relação aos aposentados e pensionistas do estudo, ao considerar que a maioria dos idosos estudados está na faixa etária entre 60 e 69 anos, é possível que alguns estejam na condição de aposentados e em plena atividade, o que pode torná-los vulneráveis aos traumas, uma vez que necessitam se deslocar para realizar atividades laborais ou exercer atividades relacionadas a práticas de risco.

Ao processo de envelhecimento podem estar associadas as doenças crônicas não transmissíveis, as quais compõem o elevado número de casos de morbimortalidade da população brasileira. Dados censitários ${ }^{(13)}$, no ano de 2008 com 59,5 miIhões de brasileiros, evidenciam que $31,3 \%$ deles afirmaram ter pelo menos uma doença crônica; entre aqueles com 65 anos ou mais, 79,1\% declararam possuir alguma doença crônica, sendo que destes, 28,3\% possuíam três ou mais doenças.

Em estudo ${ }^{(5)}$ com pacientes acima de 15 anos, vítimas de trauma, e com diagnóstico de doença isquêmica do coração, cirrose, coagulopatia congênita, doença pulmonar obstrutiva crônica ou diabetes, estas foram consideradas importantes fatores preditivos da mortalidade.

A HAS, observada neste estudo, pode gerar consequências negativas na condição respiratória e hemodinâmica do 
paciente após o trauma, antecedendo estados de hipóxia e hipoperfusão, podendo até confundir no momento da avaliação da gravidade do trauma, pois a resposta hemodinâmica é limitada nesta população(14) e o valor da pressão arterial apresentada no idoso hipertenso pode ser considerado normal, quando na verdade, já se inicia um quadro de hipovolemia ${ }^{(14)}$.

Deve-se considerar que, nestes casos, podem ocorrer alterações cardíacas e/ou renais, demandando atenção especial no controle da diurese e o exame frequente dos aparelhos cardiovascular e respiratório do idoso, com vistas à prevenção, por exemplo, de falência ventricular esquerda durante estados de choque ${ }^{(4)}$.

Verificou-se, neste estudo, a média de 2,3 doenças preexistentes por idoso. A esse respeito, é comum a presença de mais de uma doença por idoso ${ }^{(5)}$ e à medida que o número de doenças aumenta, há maiores chances de óbito após o trauma ${ }^{(15)}$.

Os anti-hipertensivos $(33,4 \%)$ predominaram dentre os medicamentos em uso pelos idosos. Em outro estudo ${ }^{(16)}$, também há evidências da utilização deles pela maioria dos idosos, vítimas de trauma, devendo-se estar alerta para o fato de que, nestes casos, a ação desta classe de medicamento pode levar à diminuição da resposta cardiovascular ao trauma, principalmente na presença do choque ${ }^{(17)}$, dificultando seu diagnóstico precoce e podendo ocasionar a morte do idoso ${ }^{(18)}$.

A este respeito, deve-se atentar que, normalmente, os idosos fazem uso, em média, de três a quatro medicamentos diferentes, muitas vezes sem controle médico, possibilitando, portanto, a ocorrência de interações medicamentosas com as drogas administradas para o tratamento cirúrgico, quando indicado ${ }^{(19)}$.

Há, também, relação entre o uso de medicamentos por idosos e a ocorrência de traumas, devido provavelmente aos efeitos adversos como hipotensão postural, sonolência, tonturas e a necessidade de urinar com maior frequência ${ }^{(20)}$.

Quanto ao tipo de transporte pré-hospitalar, deve-se considerar, durante o atendimento inicial, a estabilização clínica dos pacientes e a gravidade do trauma, a fim de que sejam transferidos para os hospitais de referência em trauma que atendam suas necessidades e, preferencialmente, após a triagem pela regulação médica. Observou-se que o transporte pré-hospitalar, por USB, principalmente de pacientes traumatizados em estado grave e procedentes de cidade vizinhas ao local de estudo, é inadequado, podendo expor a vítima à morte ${ }^{(21)}$.

Evidenciou-se o predomínio de quedas (31,3\%), seguidas por atropelamentos $(28,2 \%)$, resultados semelhantes aos encontrados na literatura ${ }^{(1)}$. A queda é considerada um problema de saúde pública e está associada a fatores de risco intrínsecos e extrínsecos. As complicações decorrentes da queda no idoso podem ser caracterizadas pela imobilização prolongada, rabdomiólise, desidratação e infecção. Além disso, acrescentam que as lesões decorrentes de quedas em pacientes geriátricos tendem a ser mais graves do que aquelas apresentadas por pacientes jovens e com quedas similares ${ }^{(6)}$.

Vale mencionar que falhas na infraestrutura física de vias públicas, tais como a falta de calçamentos e rampas de acesso a prédios públicos, obstáculos nas calçadas, entre outros, dificultam o livre trânsito do idoso e aumentam o risco de quedas. Além disso, fatores como estes dificultam a acessibilidade do idoso aos diversos setores e atividades sociais e, por conseguinte, sua inserção social ${ }^{(22)}$.

Os idosos, com facilidade, são vítimas de atropelamentos, em especial na presença de largas avenidas que nem sempre Ihes permite a travessia em tempo hábil e programado pelos semáforos, mesmo utilizando a faixa de pedestres ${ }^{(23)}$.

Geralmente, idosos condutores de veículos, ao se envolverem em acidentes, estão em baixas velocidades, não alcoolizados e próximos de suas residências ${ }^{(22)}$, sendo os homens mais frequentemente vítimas na condição de condutores e, as mulheres, na de passageiras ${ }^{(1)}$.

Quanto aos índices de trauma, considerando as regiões do corpo afetadas, destaca-se a cabeça/pescoço seguida pelas extremidades e ossos da pelve. Deve-se levar em consideração o mecanismo do trauma ao se verificar a localização das lesões nos diversos segmentos corpóreos ${ }^{(7)}$.

O segmento cefálico pode ser lesionado tanto pelo impacto contra o veículo quanto contra o solo, porém, a maior gravidade das lesões está relacionada ao impacto contra o veículo. O para-brisa parece ser a estrutura do automóvel causadora das lesões mais graves sobre o segmento cefálico. Da mesma forma, fraturas dos membros inferiores são comuns e parecem ser decorrentes da associação da angulação e rotação dos membros inferiores ${ }^{(24)}$.

Em relação à gravidade das lesões, as de extremidades são mais frequentes, porém os maiores escores estão presentes no segmento cefálico, podendo acarretar hemorragias intracranianas e contusões cerebrais ${ }^{(25)}$. Uma consequência irreversível observada nestas condições é a maior probabilidade de óbito ${ }^{(26)}$.

Os valores obtidos pelo RTS e ECGI para os idosos pesquisados indicam melhores perspectivas de recuperação e melhor qualidade de vida para a maioria deles após o evento traumático. Ao demonstrarem graus variados de comprometimento no paciente, estas escalas atuam de forma a direcionar o atendimento ${ }^{(7)}$, conforme sua complexidade, proporcionando sua continuidade e evitando danos adicionais.

Quanto à gravidade do trauma, foi possível verificar que estudos que abordam traumas em idosos evidenciam pontuação variada para o ISS, relacionando-o com a faixa etária ou com o mecanismo do trauma ${ }^{(27)}$. No geral, verifica-se que a gravidade calculada pelo ISS é relativamente baixa para idosos; contudo, suas implicações atingem maiores proporções, principalmente, no que diz respeito à probabilidade de mor$\mathrm{te}^{(28)}$. Houve alta chance de sobrevida calculada pelo TRISS, no entanto, deve-se ter cautela ao utilizar isoladamente estes índices para a análise da evolução do trauma no idoso, pois fatores como o sexo, a idade e estado patológico parecem ser igualmente importantes ${ }^{(1)}$.

Em se tratando das associações realizadas neste estudo, é possível supor que a causa dos traumas em idosos pesquisados seja orgânica ou patológica para as mulheres, e acidental para os homens. A este respeito medidas preventivas específicas para o trauma se fazem necessárias.

Quanto ao desfecho, nota-se que as extremidades foram a segunda região do corpo mais acometida e a primeira em necessidade cirúrgica. Este percentual elevado de procedimentos cirúrgicos realizados, talvez se justifique pelo fato 
de que o local de estudo seja reconhecido como serviço de referência para o tratamento de lesões ortopédicas. Pode-se inferir também, que o mecanismo e a gravidade do trauma predispuseram os idosos às fraturas e, assim, com necessidade de abordagem cirúrgica para o tratamento.

As lesões em cabeça/pescoço acometeram grande parte dos idosos desta pesquisa que, consequentemente, foi a região do corpo considerada como maior causadora dos óbitos. Alguns fatores estão associados aos piores prognósticos deste tipo de lesão, tais como: idades mais avançadas, menores pontuações na ECGI e ausência de reatividade pupilar ${ }^{(26)}$, a presença de doenças, o uso de anticoagulantes e anti-inflamatórios ${ }^{(29)}$.

Os resultados deste estudo destacaram que idosos jovens foram vítimas de acidentes, enquanto idosas mais velhas, de quedas. Houve predomínio do trauma moderado, sendo a região da cabeça/pescoço a parte do corpo mais acometida, com lesão grave, porém sem risco de vida; mesmo assim, parte significativa evoluiu a óbito. Mediante os dados aqui expostos e com vistas a evitar piores resultados, faz-se necessária a elaboração de medidas preventivas e curativas do trauma em idosos. Neste sentido, recomenda-se monitoração invasiva precoce na tentativa de reduzir complicações diversas e aumentar as chances de sobrevida desta população ${ }^{(15)}$, ao considerar que a história patológica pregressa e o consequente uso de medicamentos podem mascarar uma condição mais gra$\mathrm{ve}^{(1)}$. Torna-se importante desenvolver pesquisas abordando a vulnerabilidade e os riscos que pedestres e motoristas idosos apresentam ao se envolverem em acidentes.

\section{CONSIDERAÇÕES FINAIS}

O presente estudo possibilitou identificar as características e evolução do trauma em idosos, a partir da análise dos dados de natureza secundária obtidos no NHE de um hospital geral terciário de uma cidade do interior paulista, entre 2008 e 2010.

Vale ressaltar que os dados aqui apresentados são peculiares para a amostra estudada, portanto generalizações devem ser evitadas.
Em decorrência das alterações biopsicossociais do processo de envelhecimento, a assistência aos pacientes geriátricos vítimas de traumas deve ser diferenciada. Além das mudanças comuns, a presença de doenças crônicas e o uso de medicamentos pré-trauma podem influenciar na reação orgânica dos pacientes senis.

Este estudo assumiu um caráter diferencial quando se optou por investigar apenas a população de idosos, vítimas de trauma, atendidos em um centro de referência para urgências e emergências.

Acredita-se que as questões abordadas nesta pesquisa poderão contribuir para a compreensão do impacto do trauma na vida do idoso, uma vez que suas consequências físicas, emocionais e sociais, podem ser evitadas, por meio de medidas preventivas que abordem a educação no trânsito, prevenção de acidentes, valorização do indivíduo em qualquer idade, cuidados com a saúde dos idosos, entre outros.

Além disso, a possibilidade de utilização de sistemas de pontuação para a avaliação das alterações fisiológicas, anatômicas e da probabilidade de sobrevida de idosos traumatizados permite uma mensuração das reais respostas do idoso ao trauma.

Apesar disso, sabe-se que estes índices apresentam algumas limitações na predição de sobrevida, especialmente em se tratando de populações específicas como os idosos que geralmente possuem reserva fisiológica diminuída, apresentam doenças prévias e fazem uso de medicamentos diversos. Dessa forma, estes fatores devem estar claros para a equipe que o assiste, sobretudo em seu primeiro atendimento, possibilitando a prevenção de complicações.

\section{AGRADECIMENTOS}

Ao Professor Doutor Afonso Diniz Costa Passos, chefe do Núcleo Hospitalar de Vigilância Epidemiológica do Hospital das Clínicas da Faculdade de Medicina de Ribeirão Preto, Universidade de São Paulo, pela autorização e disponibilidade dos dados utilizados nesta pesquisa.

\section{REFERÊNCIAS}

1. Souza JAG, Iglesias ACRG. Trauma no idoso. AMB Rev Assoc Med Bras. 2002;48(1):79-86.

2. Souza RKT, Soares DFPP, Mathias TAF, Andrade OG, Santana RG. Idosos vítimas de acidentes de trânsito: aspectos epidemiológicos. Acta Sci Health Sci [Internet]. 2003;25(1):19-25. Disponível em: http://periodicos.uem. br/ojs/index.php/ActaSciHealthSci/article/view/2247/0

3. Ministério da Saúde (BR), Departamento de Informática do Sistema Único de Saúde. Epidemiologias e morbidades. Brasília (DF): Ministério da Saúde; 2012.

4. Freire E. Trauma: a doença dos séculos. São Paulo (SP): Atheneu; 2001.

5. Morris JÁ Jr, Mackenzie EJ, Eldelstein SL. The effects of preexisting conditions on mortality in trauma patients.
JAMA. 1990;263(14):1942-6.

6. Aschkenasy MT, Rothenhaus TC. Trauma and falls in the elderly. Emerg Med Clin North Am. 2006; 24(2):413-32.

7. Pereira Júnior GA, Scarpelini S, Basile-Filho A, Andrade JI. Índices de trauma. Medicina (Ribeirão Preto). 1999;32(3):237-50.

8. Ribeiro AP, Souza ER, Atie S, Souza AC, Schilith AO. A influência das quedas na qualidade de vida dos idosos. Ciênc Saúde Coletiva [Internet] 2008 [acesso em 19 de abril de 2013];13(4):1265-73. Disponível em: http:// www.scielo.br/pdf/csc/v13n4/23.pdf

9. Bergeron E, Clement J, Lavoie A, Ratte S, Bamvita JM, Aumont $F$, et al. A simple fall in the elderly: not so simple. J Trauma. 2006;60(2):268-73. 
10. Universidade de São Paulo (BR), Faculdade de Medicina de Ribeirão Preto Departamento de Medicina Social. Doenças e agravos não transmissíveis - DANT, Ribeirão Preto-SP e região. Ribeirão Preto (SP): Centro de Processamento de Dados Hospitalares; 2010.

11. Silveira R, Rodrigues RAP, Costa Júnior ML. Idosos que foram vítimas de acidentes de trânsito no município de Ribeirão Preto-SP, em 1998. Rev Latinoam Enferm. 2002;10(6):765-71.

12. Souza ER. Masculinidade e violência no Brasil. Ciênc Saúde Coletiva [Internet]. 2005 [acesso em 19 de abril de 2013];10(1):59-70. Disponível em: http:// www.scielo.br/scielo.php?script =sci_arttext\&pid $=$ S1413-81232005000100012

13. Instituto Brasileiro de Geografia e Estatística. Pesquisa Nacional por Amostra de Domicílios. Um panorama da saúde no Brasil: acesso e utilização dos serviços, condições de saúde e fatores de risco e proteção à saúde. Rio de Janeiro (RJ): IBGE; 2010.

14. ParreiraJG, SoldáSC, PerlingeiroJAG, PadoveseCC, Karakhanian WZ, AssefJC. Análise comparativa das características do trauma em pacientes idosos e não idosos. Rev AssocMed Bras [Internet]. 2010 [acesso em 19 de abril de 2013];56(5):5416. Disponível em: http://www.scielo.br/scielo.php?pid=S0 104-42302010000500014\&script = sci_arttext

15. Ferrera PC, Bartfield JM, D'Andrea CC. Geriatric trauma: outcomes of elderly patients discharged from the ED. Am J Emerg Med. 1999;17(7):629-32.

16. Hamra A, Ribeiro MB, Miguel OF. Correlação entre fratura por queda em idosos e uso prévio de medicamentos. Acta Ortop Bras. 2007;15(3):143-5.

17. Neideen T, Lam M, Brasel KJ. Preinjury beta blockers are associated with increased mortality in geriatric trauma patients. J Trauma. 2008;65(5):1016-20.

18. Rubenstein LZ. Falls in older people: epidemiology, risk factors and strategies for prevention. Age Ageing [Internet]. 2006 [cited 2013 April 19];35(52):37-41. Available from: http://ageing.oxfordjournals.org/content/35/suppl_2 /ii37.abstract

19. Silva EDO, Gomes ACA, Gomes DO, Vianna K, Melo P. Trauma no idoso. Rev Cir Traumat Buco-Maxilo-Facial [Internet]. 2001 [acesso em 19 de abril de 2013];1(2):7-12. Disponível em: http://www.revistacirurgiabmf.com/2001/ v1n2/v1n2.1.pdf

20. Camilloni L, Farchi S, Rossi PG, Chini F, Biorgia P. Mortality in elderly injured patients: the role of comorbidities. Int J Inj Contr Saf Promot [Internet]. 2008 [cited 2013
April 19];15(1):25-31. Available from: http://www.ncbi. nlm.nih.gov/pubmed/18344093

21. Pereira Júnior GA, Scarpelini S, Aquino AMF, Santiago RC, Negrini BVM, Passos ADC. Caracterização dos pacientes traumatizados atendidos na Unidade de Emergência do Hospital das Clínicas de Ribeirão Preto da Universidade de São Paulo utilizando os índices de gravidade RTS, ISS e TRISS em 2006/2007. Boletim Epidemiológico Paulista. 2009;6(62):4-11.

22. Fernandes JC. Urbanismo e envelhecimento: algumas reflexões a partir da cidade de Uberlândia. Rev Caminhos Geografia [Internet]. 2000 [acesso em 19 de abril de 2013];1(2):31-49. Disponível em: http://www. seer.ufu.br/index.php/caminhosdegeografia/article/ viewFile/15252/8553

23. Sant'Anna RM, Braga MGC, Santos MPS. Segurança no trânsito para os motoristas idosos: desafios e perspectivas. Textos Envelhecimento [Internet]. 2004 [acesso em 19 de abril de 2013];7(1):1-12. Disponível em: http://revista.unati.uerj.br/scielo.php?script =sci_arttex t\&pid =S1517-59282004000100002\&lng = pt\&nrm = isso

24. Paranhos WY. Trauma no idoso. In: Sousa RMC, Calil AM, Paranhos WY, Malvestio MA. Atuação no trauma: uma abordagem para a enfermagem. São Paulo (SP): Atheneu; 2009. p.411-7.

25. Patel HC, Bouamra O, Woodford M, Yates DW, Lecky FE. Clinical article: mortality associated with severe head injury in the elderly. Acta Neurochir. 2010;152(8):1353-7.

26. MRC CRASH Trial Collaborators, Perel P, Arango M, Clayton T, Edwards P, Komolafe E, et al. Predicting outcome after traumatic brain injury: pratical prognostic models based on large cohort of international patients. BMJ [Internet]. 2008 [cited 2013 April 19];336(7641):425-9. Available from: http://www.ncbi.nlm.nih.gov/pubmed/18270239

27. Smith DP, Enderson BL, Maull KI. Trauma in the elderly: determinants of outcome. Souther Med J [Internet]. 1990 [cited 2013 April 19];83(2):171-7. Available from: http:// www.ncbi.nlm.nih.gov/pubmed/2305297

28. Van der Sluis CK, Timmer HW, Eisma WH, Ten Dius HJ. Outcome in elderly injured patients: injury severity versus host factors. Injury. 1997;28(9-10):588-92.

29. Braga FM, Adam-Netto A, Santos ER, Braga PB. Avaliação dos 76 casos de TCE por queda da própria altura atendidas na emergência de um hospital geral. ACM Arq Catarin Med [Internet] 2008 [acesso em 19 de abril de 2013];37(4):35-39. Disponível em: http://www.acm.org. br/revista/pdf/artigos/608.pdf 